\title{
Endoscopia respiratória em 89 pacientes com neoplasia pulmonar
}

\author{
PAUlo José LORENZONI ${ }^{1}$, MARIA IlizABETI DONATTI ${ }^{2}$ \\ Paulo de TARso MulleR ${ }^{3}$, Pedro NANGo DOBASHI ${ }^{4}$
}

Objetivos: As neoplasias pulmonares são uma das mais freqüentes causas de mortalidade no Brasil. Objetivou-se com o presente estudo avaliar o diagnóstico dessas neoplasias através da fibrobroncoscopia. Método: Foram analisados, retrospectivamente, 89 pacientes no período de cinco anos, divididos em dois grupos: grupo $1(\mathrm{n}=53)$ - presença de lesão visível à endoscopia, grupo 2

$(n=36)$ - ausência de lesões na endoscopia. Na revisão dos prontuários retiraram-se os dados epidemiológicos, os achados endoscópicos, os exames cito e anatomopatológicos, bem como o exame que proporcionou o diagnóstico final. Resultados: Os achados endoscópicos indiretos de neoplasia corresponderam a 59,5\% e a presença de tumor na luz brônquica a 48,8\%. O tipo histológico mais freqüente foi o carcinoma epidermóide $(59,5 \%)$, seguido do adenocarcinoma $(15,7 \%)$, carcinoma

indiferenciado de pequenas células $(10,1 \%)$ e outros $(14,7 \%)$. Nos tumores visíveis ou não na endoscopia o estudo anatomopatológico da biópsia brônquica e/ou da citopatologia do lavado e do escovado brônquico mostrou sensibilidade de $83,1 \%$ no diagnóstico de neoplasia pulmonar. Quando a lesão era visível à inspeção endoscópica, a sensibilidade atingiu percentual de $96,2 \%$, sendo a biópsia positiva em $94,2 \%$ e a citologia em 43,75\%. Quando não se observava lesão brônquica o rendimento foi sensivelmente menor (63,8\%): a biópsia mostrou percentual de $72,7 \%$ e a citopatologia de $38,4 \%$.

As complicações menores decorrentes do exame endoscópico ocorreram em 11,2\% dos pacientes; não ocorreram complicações graves. Conclusão: A fibrobroncoscopia foi um excelente método para investigação de pacientes com suspeita de neoplasia pulmonar com sensibilidade de $83,1 \%$; sua sensibilidade foi maior nas lesões visiveis. Nas lesões não visíveis a sensibilidade aumentou com a biópsia endobrônquica. (J Pneumol 2001;27(2):83-88)

\section{Respiratory endoscopy in 89 patients of lung neoplasms}

Objectives: Lung neoplasms are one of the most common causes of mortality in Brazil. The objective of this survey is to evaluate the diagnosis of these neoplasms using fiberoptics bronchoscopy. Methods: 89 patients were analyzed retrospectively in a period of five years. They were divided into two groups: Group $1(n=53)$ - the presence of visible lesion on endoscopy,

Group $2(n=36)$ - the absence of visible lesion on endoscopy. Epidemiological elements, endoscopic discoveries, cytopathologic and anatomopathologic examination were performed in the review, as well the examination that provided the final diagnosis. Results: The findings of indirect endoscopies of neoplasms corresponded to $59.5 \%$ and the presence of tumor inside bronchi was $48.8 \%$. The most frequent histologic type was the epidermoid carcinoma (59.5\%),

followed by adenocarcinoma (15.7\%), small-cell carcinoma (10.1\%), and others (14.7\%). In endoscopically visible or not visible tumors, the anatomopathologic study of bronchial biopsy and/ or the cytopathologic examination of the bronchial washing and brushing showed a sensitivity of

* Trabalho realizado na Disciplina de Pneumologia, Departamento de Clínica Médica, Universidade Federal de Mato Grosso do Sul (UFMS).

1. Médico Residente de Clínica Médica do Hospital Universitário UFMS.

2. Médica Pneumologista da FNS/Hospital Universitário - UFMS.

3. Professor Assistente da Disciplina de Pneumologia, Departamento de Clínica Médica, Universidade Federal de Mato Grosso do Sul.
4. Professor Titular da Disciplina de Pneumologia, Departamento de Clínica Médica, Universidade Federal de Mato Grosso do Sul.

Endereço para correspondência - Paulo José Lorenzoni, Rua Francisco Alves Castelo, 306, Ap. 17 - 79080-770 - Campo Grande, MS, Tel: (67) 746-6189 - e-mail pjlorenzoni@bol.com.br.

Recebido para publicação em 3/3/00. Reapresentado em 11/7/00. Aprovado, após revisão, em 25/8/00. 
$83.1 \%$ in the diagnosis of lung neoplasms. When the lesion was visible on endoscopy, the sensitivity was $96.2 \%$ : positive biopsy was $94.2 \%$, and cytology was $43.75 \%$. When the lesion in the bronchi is not observed, its addition was significantly small (63.8\%), namely: biopsy registered

72.7\% and cytopathology $38.4 \%$. Not significant complications resulting from the endoscopic examination occurred in $11.2 \%$ of patients and there were no serious complications. Conclusion:

The fiberoptics bronchoscopy was an excellent method to investigate patients with suspicion of

lung neoplasms, with $83.1 \%$ of sensitivity. This sensitivity was higher in visible lesions and in non-visible lesions when endobronchial biopsy was used.

Descritores - Neoplasias pulmonares. Broncoscopia. Carcinoma broncogênico. Técnicas de diagnóstico e procedimentos.

Key words - Lung neoplasms. Bronchoscopy. Bronchogenic carcinoma. Diagnostic techniques and procedures.

\section{INTRODUÇ̃̃O}

A incidência do câncer de pulmão no Brasil tem aumentado nas últimas décadas ${ }^{(1-4)}$ e, apesar dos consideráveis avanços nas técnicas diagnósticas e modalidades de tratamento, sua mortalidade continua aumentando em todo o mundo(4). No Mato Grosso do Sul, os tumores malignos constituem a nona maior causa de mortalidade $e^{(5)}$, sendo a neoplasia pulmonar a principal causa de morte por tumor maligno no sexo masculino e a segunda dentre as neoplasias do sexo feminino.

Tendo em vista esse alarmante indice de mortalidade por neoplasia pulmonar, em ambos os $\operatorname{sexos}^{(3-6)}$, faz-se necessário que seu diagnóstico seja precoce.

No tocante ao diagnóstico do câncer de pulmão é de extrema importância definir o tipo histológico e estadiamento ${ }^{(7)}$. Isso faz com que exames complementares tenham um papel de destaque no manejo dos pacientes com essa doença.

A fibrobroncoscopia é, de maneira geral, o exame que apresenta o melhor potencial diagnóstico em carcinoma brônquico. É um procedimento seguro, que possibilita não só a visualização da lesão, como também a colheita de material para citologia e histologia (lavado e escovado brônquico, biópsias endo e transbrônquicas), tanto para confirmação diagnóstica, como para seu estadiamento ${ }^{(3,4,8-13)}$.

$\mathrm{O}$ desenvolvimento dos fibrobroncoscópios iniciou-se a partir de 1966 com Shigeto Ikeda ${ }^{(14)}$ e possibilitou investigação mais aprofundada da árvore brônquica, com melhor tolerância pelo paciente $e^{(3,4,14,15)}$. O acesso aos lobos superiores e a colheita de material para exames laboratoriais e anatomopatológicos passaram a proporcionar melhor diagnóstico em muitas lesões pulmonares, especialmente no carcinoma brônquico.

Vários estudos têm descrito formas diferentes de abordar o paciente com neoplasia pulmonar por fibrobroncoscopia, fazendo com que resultados diferentes sejam obtidos pelo exame endoscópico num mesmo grupo de pa- cientes. No diagnóstico da neoplasia pulmonar por fibrobroncoscopia, as diversas séries da literatura mostram sensibilidades que variam de $48 \%$ a $95 \%$, conforme o método cito ou anatomopatológico utilizado e a lesão ser endoscopicamente visivel ou não(6,8,12,16,17).

Essa variação de sensibilidade tornou relevante a necessidade de avaliar como tem sido realizado o diagnóstico de neoplasia pulmonar por fibrobroncoscopia, no Setor de Endoscopia Respiratória do Serviço de Pneumologia do Hospital Universitário da Universidade Federal de Mato Grosso do Sul (HU-UFMS). Devido ao crescimento da incidência da neoplasia pulmonar o diagnóstico precoce, utilizando o exame mais adequado para tanto e os benefícios trazidos pelo desenvolvimento das técnicas utilizadas na fibrobroncoscopia, permitirá que mais doentes sejam curados. Hoje a investigação com fibrobroncoscopia pode atingir locais mais profundos do pulmão e, ainda, com menores incômodos para o paciente.

\section{MÉTOdO}

No período de janeiro/1994 a dezembro/1998 foram realizadas 1.033 fibrobroncoscopias por diversos motivos, em nível ambulatorial, no Setor de Endoscopia Respiratória do Serviço de Pneumologia do Hospital Universitário da Universidade Federal de Mato Grosso do Sul (HU-UFMS).

Foram identificados 89 pacientes com câncer de pulmão. A fibrobroncoscopia proporcionou o diagnóstico em $74(83,1 \%)$ pacientes, enquanto nos 15 restantes o diagnóstico final foi estabelecido por outros métodos, tais como: biópsia a céu aberto, punção transcutânea e citologia do líquido pleural. Os 89 pacientes constituem a amostra estudada e foram divididos em dois grupos: grupo 1, composto de 53 pacientes com tumor endoscopicamente visivel; grupo 2 , composto de 36 indivíduos com tumor endoscopicamente não visível.

Foi definido por Ikeda ${ }^{(8,14,18)}$, como endoscopicamente visivel, quando se visualiza o tumor e/ou obstrução e/ou necrose tumoral, bem como sinais de infiltração caracterizados por: hiperemia da mucosa, ingurgitamento dos vasos submucosos e desaparecimento da impressão dos anéis cartilaginosos. Quando nenhum desses achados está presente ou somente há sinais indiretos de tumor (como carinas rombas e compressão extrínseca) ou, ainda, quando 
os critérios de infiltração eram incompletos, o tumor é classificado como endoscopicamente não visivel ${ }^{(8,14,18)}$.

Da revisão dos prontuários, retiravam-se dados epidemiológicos, achados endoscópicos, exames cito e anatomopatológicos, bem como o método que proporcionou o diagnóstico final. Para fins de cadastramento considerouse diagnóstico definitivo quando havia confirmação cito ou anatomopatológica da neoplasia pulmonar.

O exame endoscópico foi realizado com fibrobroncoscópios da marca Olympus, modelos BF type P20D ou $B F$ type XT20. Com a suspeita clínico-radiológica de neoplasia pulmonar, era indicado o exame. O paciente era conduzido ao centro endoscópico após seis horas de jejum. Não houve necessidade de sedação. A anestesia tópica de via aérea superior (narina) foi realizada com lidocaína gel a $2 \%$, orofaringe com spray de lidocaína a $10 \%$; e de via aérea inferior (árvore brônquica) com lidocaína a $2 \%$, pelo broncoscópio (dose máxima $20 \mathrm{ml}$ ). O fibrobroncoscópio foi introduzido pela narina direita ou esquer$\mathrm{da}$, com o paciente preferencialmente em decúbito dorsal.

As 1.033 fibrobroncoscopias foram realizadas por um dos três endoscopistas do Serviço de Pneumologia do HUUFMS. Procedia-se à visualização da árvore brônquica, iniciando pelo lado contralateral à lesão. Nos pacientes com lesões visiveis, realizaram-se lavado, escovado e biópsia. Nas biópsias endobrônquicas, na maioria dos pacientes retiravam-se três fragmentos do mesmo local. Nas lesões não visíveis, havendo presença de sinais indiretos de tumor ou critérios incompletos de infiltração, realizavam-se o lavado, escovado e a biópsia brônquica (sem fluoroscopia). Quando não havia qualquer achado broncoscópico de lesão tumoral realizavam-se lavado e escovado no brônquio do segmento correspondente à localização radiológica. O lavado broncoalveolar foi realizado encaixando o fibrobroncoscópio em um brônquio o mais distal possivel e administrando $120 \mathrm{ml}$ de soro fisiológico, sendo recuperados cerca de $50 \%$ do material ${ }^{(14,15)}$. Nesse grupo não foram realizadas biópsias transbrônquicas.

O diagnóstico de certeza de câncer foi aceito quando o exame citopatológico do lavado ou do escovado brônquico e/ou do anatomopatológico demonstrava alterações compativeis com neoplasia pulmonar.

\section{RESULTADOS}

A amostra de 89 indivíduos foi dividida em: grupo 1 , composto de $53(59,5 \%)$ pacientes com tumor endoscopicamente visivel; grupo 2 , com $36(40,5 \%)$ pacientes com tumor endoscopicamente não visivel. Idade média foi de 60,8 anos, sendo $61(68,5 \%)$ homens e $28(31,5 \%) \mathrm{mu}-$ lheres. Dos 89 pacientes, 61 (68,5\%) eram tabagistas. Esses parâmetros, quando comparados entre os dois grupos, mostraram predomínio de homens (4:1) no grupo 1 , em relação ao grupo 2 (1:1).

\section{Achados endoscópicos}

Grupo 1: Na inspeção endoscópica, 43 pacientes $(81,1 \%)$ apresentavam tumor na luz brônquica; 24 $(45,2 \%)$, sinais indiretos de tumor; dois $(3,7 \%)$, paralisia da prega vocal; um $(1,8 \%)$, ausência de alterações endoscópicas (Tabela 1).

Grupo 2: Encontramos as seguintes alterações: 29 $(80,5 \%)$ sinais indiretos de tumor, dois $(5,5 \%)$ com paralisia da prega vocal, dois $(5,5 \%)$ com sangramento brônquico e sete $(19,4 \%)$ não apresentavam anormalidades nas vias aéreas (Tabela 1 ).

Não se computaram achados inespecíficos: hiperemia e/ou edema e/ou secreção.

\section{Tipos histológicos}

Grupo 1: Em 35 (66\%) pacientes os tumores eram carcinomas epidermóides; em quatro (7,5\%), adenocarcinomas, em sete $(13,2 \%)$, indiferenciado de pequenas células; em três $(5,6 \%)$, indiferenciado de grandes células; em um $(1,8 \%)$, carcinoma in situ; em um $(1,8 \%)$, leiomioma; e em dois $(3,7 \%)$, carcinoma indiferenciado (tipo histológico não foi determinado) (Tabela 2).

Grupo 2: Em 18 (50\%) pacientes os tumores eram carcinomas epidermóides, em dez (27,7\%), adenocarcinomas, em dois $(5,5 \%)$, indiferenciado de pequenas células, em um $(2,7 \%)$, indiferenciado de grandes células, em um $(2,7 \%)$, fibroma, e em quatro $(11,1 \%)$, carcinoma indiferenciado (Tabela 2).

\section{Método que proporcionou o diagnóstico final}

Grupo 1: O diagnóstico foi estabelecido por fibrobroncoscopia em 51 casos $(96,2 \%)$ e em dois $(3,7 \%)$, por biópsia a céu aberto (Tabela 3).

Grupo 2: O diagnóstico foi estabelecido por fibrobroncoscopia em 23 casos (63,8\%); em cinco (13,8\%), por citologia pleural; em três $(8,3 \%)$, por punção transtorácica; e em cinco (13,8\%), por biópsia a céu aberto (Tabela 3).

\section{Sensibilidade da fibrobroncoscopia no diagnóstico da neoplasia pulmonar}

Grupo 1: Foram realizadas 52 biópsias, sendo 49 $(94,2 \%)$ positivas para carcinoma. O citopatológico foi

TABELA 1

Freqüência dos achados endoscópicos em pacientes com neoplasia pulmonar

\begin{tabular}{lccr}
\hline \multicolumn{1}{c}{ Achado endoscópico } & $\begin{array}{c}\text { Grupo 1 } \\
(\mathbf{n = 5 3 )}\end{array}$ & $\begin{array}{c}\text { Grupo 2 } \\
(\mathbf{n}=\mathbf{3 6})\end{array}$ & $\begin{array}{c}\text { Total } \\
(\mathbf{n = 8 9})\end{array}$ \\
\hline Tumor na luz brônquica & $43(81,1 \%)$ & 0 & $43(48,8 \%)$ \\
Sinais indiretos de tumor & $24(45,2 \%)$ & $29(80,5 \%)$ & $53(59,5 \%)$ \\
Paralisia da prega vocal & $2(3,7 \%)$ & $2(5,5 \%)$ & $4(4,4 \%)$ \\
Sangramento brônquico & 0 & $2(5,5 \%)$ & $2(2,2 \%)$ \\
Sem alterações & $1(1,8 \%)$ & $7(19,4 \%)$ & $8(8,9 \%)$ \\
\hline
\end{tabular}


realizado em 16 pacientes, sendo positivo em sete (43,7\%). Tanto a biópsia como o citopatológico foram positivos em cinco $(35,7 \%)$ e negativos em um $(7,1 \%)$ de 14 pacientes em que ambos os procedimentos foram realizados simultaneamente. Portanto, a sensibilidade da fibrobroncoscopia (citológico e/ou anatomopatológico) foi de 51 em 53 $(96,2 \%)$ (Tabela 4).

TABELA 2

Incidência dos tipos histológicos em pacientes com neoplasia pulmonar

\begin{tabular}{lccc}
\hline Tipos histológicos & $\begin{array}{c}\text { Grupo 1 } \\
(\mathbf{n = 5 3 )}\end{array}$ & $\begin{array}{c}\text { Grupo 2 } \\
(\mathbf{n = 3 6 )}\end{array}$ & $\begin{array}{c}\text { Total } \\
(\mathbf{n = 8 9})\end{array}$ \\
\hline $\begin{array}{l}\text { Carcinoma } \\
\text { epidermóide }\end{array}$ & $35(66 \%)$ & $18(50 \%)$ & $53(59,5 \%)$ \\
$\begin{array}{l}\text { Adenocarcinoma } \\
\text { Indiferenciado de }\end{array}$ & $4(7,5 \%)$ & $10(27,7 \%)$ & $14(15,7 \%)$ \\
pequenas células & $7(13,2 \%)$ & $2(5,5 \%)$ & $9(10,1 \%)$ \\
$\begin{array}{l}\text { Indiferenciado de } \\
\text { grandes células }\end{array}$ & $3(5,6 \%)$ & $1(2,7 \%)$ & $4(4,4 \%)$ \\
$\begin{array}{l}\text { Carcinoma in situ } \\
\text { Leiomioma }\end{array}$ & $1(1,8 \%)$ & 0 & $1(1,1 \%)$ \\
$\begin{array}{l}\text { Fibroma } \\
\begin{array}{l}\text { Indiferenciado } \\
\text { (não determinado) }\end{array}\end{array}$ & $1(1,8 \%)$ & 0 & $1(1,1 \%)$ \\
\hline
\end{tabular}

TABELA 3

Incidência do método que proporcionou o diagnóstico final em pacientes com neoplasia pulmonar

\begin{tabular}{lccc}
\hline Método & $\begin{array}{c}\text { Grupo 1 } \\
(\mathbf{n = 5 3})\end{array}$ & $\begin{array}{c}\text { Grupo 2 } \\
(\mathbf{n = 3 6})\end{array}$ & $\begin{array}{c}\text { Total } \\
(\mathbf{n = 8 9})\end{array}$ \\
\hline Fibrobroncoscopia & $51(96,2 \%)$ & $23(63,8 \%)$ & $74(83,1 \%)$ \\
Citologia do & 0 & $5(13,8 \%)$ & $5(5,6 \%)$ \\
$\begin{array}{l}\text { líquido pleural } \\
\text { Punção transcutânea }\end{array}$ & 0 & $3(8,3 \%)$ & $3(3,3 \%)$ \\
Biópsia por toracotomia & $2(3,7 \%)$ & $5(13,8 \%)$ & $7(7,8 \%)$ \\
\hline
\end{tabular}

TABELA 4

Sensibilidade dos procedimentos de coleta utilizados na fibrobroncoscopia (FBC) de pacientes com neoplasia pulmonar $(n=89)$

\begin{tabular}{llccccc}
\hline & Biópsia & Citologia & $\begin{array}{c}\text { Ambos } \\
\text { positivos }\end{array}$ & $\begin{array}{c}\text { Ambos } \\
\text { negativos }\end{array}$ & $\begin{array}{c}\text { Sensibilidade } \\
\text { da FBC }\end{array}$ \\
\hline \multirow{2}{*}{ Grupo 1 } & Procedimento & 52 & 16 & 14 & 14 & 53 \\
& Positivo & 44 & 2 & 5 & 1 & 51 \\
& Sensibilidade positiva & $84,6 \%$ & $12,5 \%$ & $35,7 \%$ & $7,1 \%$ & $96,2 \%$ \\
\multirow{2}{*}{ Grupo 2 } & Procedimento & 22 & 26 & 13 & 13 & 36 \\
& Positivo & 13 & 7 & 3 & 3 & 23 \\
\multirow{2}{*}{ Total } & Sensibilidade positiva & $59 \%$ & $26,9 \%$ & $23 \%$ & $23 \%$ & $63,8 \%$ \\
& & $57 / 74$ & $9 / 42$ & $8 / 27$ & $4 / 27$ & $74 / 89$ \\
& & $77 \%$ & $21,4 \%$ & $29,6 \%$ & $14,8 \%$ & $83,1 \%$ \\
\hline
\end{tabular}

Grupo 2: Foram realizadas 22 biópsias endobrônquicas, sendo $16(72,7 \%)$ positivas para carcinoma. O citopatológico foi realizado em 26 pacientes, sendo positivo em dez (38,4\%). Ambos foram negativos em três (23\%) e em três (23\%) ambos foram positivos nos 13 pacientes em que ambos os procedimentos foram simultaneamente realizados. Considerando-se a demonstração de neoplasia por ambos os métodos, a sensibilidade foi de 23/36, ou seja, 63,8\% (Tabela 4).

A sensibilidade global da fibrobroncoscopia foi de $83,1 \%$, sendo diagnosticados 74 casos de neoplasia de um total de 89 pacientes (Tabela 4).

\section{Complicações da broncoscopia}

Apenas dez (11,2\%) dos pacientes apresentaram complicações decorrentes do exame endoscópico, sendo em cinco $(5,6 \%)$, sangramento brônquico; em quatro $(4,4 \%)$, broncoespasmo; em um (1,1\%), broncorréia.

Grupo 1: Em 15\% dos pacientes houve alguma complicação do exame endoscópico, sendo: em 7,5\%, sangramento brônquico; em 5,6\%, broncoespasmo; e em 1,8\%, broncorréia (Tabela 5).

Grupo 2: Ocorreram complicações em $5,5 \%$ dos pacientes, sendo: em $2,7 \%$, sangramento brônquico e em 2,7\%, broncoespasmo (Tabela 5).

\section{DisCUSSÃO}

Analisamos retrospectivamente 89 pacientes com diagnóstico de neoplasia pulmonar, confirmada por exame citológico ou histológico, submetidos à fibrobroncoscopia no Setor de Endoscopia Respiratória do Serviço de Pneumologia do HU-UFMS. Os pacientes pertenciam a um hospital geral e a indicação do exame coube ao médico-assistente, frente à hipótese diagnóstica de neoplasia pulmonar. Tal enfoque permitiu evitar tendência à seleção de subgrupos de pacientes advindos de serviços especializados e à supervalorização do exame endoscópico.

A divisão da amostra em dois grupos, um com tumores visíveis e outro sem evidências diretas de tumor, é fundamental para análise, já que a sensibilidade da fibrobroncoscopia nas diversas séries publicadas, nacional $e$ internacionalmente, é distinta para cada grupo (Tabela 6).

Como se observa na Tabela 2 , no grupo de lesões visiveis, $66 \%$ dos tumores foram carcinomas epidermóides, semelhante ao grupo 2, que foi de $50 \%$. No entanto, o adenocarcinoma predominou no gru- 
po 2 , que foi de $27,7 \%$ contra $7,5 \%$ no grupo 1 , enquanto o carcinoma indiferenciado de pequenas células predominou no grupo 1 com $13,2 \%$ e $5,5 \%$ no grupo 2 . Esses dados concordam com vários autores ${ }^{(4,8,19-23)}$, que em suas séries descrevem carcinomas epidermóides e indiferenciado de pequenas células como lesões visiveis à endoscopia por serem mais centrais. Já os adenocarcinomas, por ser mais periféricos ${ }^{(4,8,22)}$, não são visiveis à endoscopia ou se apresentam como sinais indiretos de tumor.

Considerando-se a amostra como um todo, o tipo histológico mais freqüente foi o carcinoma epidermóide, em $59,5 \%$ dos casos. Esse valor está próximo ao limite de variação de $43 \%$ a $60 \%$, descrito por vários autores $^{(4,6,8,9,14,16)}$. O segundo tipo mais freqüente foi o adenocarcinoma, em $15,7 \%$, percentual semelhante aos relatados em outras séries $(6,8,9,14,16)$.

Os achados endoscópicos indiretos de neoplasia predominaram na presente série, correspondendo a $59,5 \%$ dos casos. A visualização direta do tumor foi o achado predominante em 48,8\%. Esta série apresentou, ainda, 8,9\% de exames broncoscópicos normais. Os tumores de origem central ou de origem periférica com centralização secundária foram predominantes.

Mak et al. ${ }^{(20)}$ descreveram a presença de tumor visivel em $57,5 \%$ de sua casuística, enquanto Ono et al. ${ }^{(21)}$, em

TABELA 5

Complicações menores decorrentes da fibrobroncoscopia em pacientes com neoplasia pulmonar

\begin{tabular}{lccc}
\hline \multicolumn{1}{c}{ Complicações } & $\begin{array}{c}\text { Grupo 1 } \\
(\mathbf{n = 5 3 )}\end{array}$ & $\begin{array}{c}\text { Grupo 2 } \\
(\mathbf{n = 3 6 )}\end{array}$ & $\begin{array}{c}\text { Total } \\
(\mathbf{n = ~ 8 9 )})\end{array}$ \\
\hline Sangramento brônquico & $4(7,5 \%)$ & $1(2,7 \%)$ & $5(5,6 \%)$ \\
Broncoespasmo & $3(5,6 \%)$ & $1(2,7 \%)$ & $4(4,4 \%)$ \\
Broncorréia & $1(1,8 \%)$ & 0 & $1(1,1 \%)$ \\
Total & $8(15,0 \%)$ & $2(5,5 \%)$ & $10(11,2 \%)$ \\
\hline
\end{tabular}

TABELA 6

Sensibilidade diagnóstica da fibrobroncoscopia nas neoplasias pulmonares em diversas séries

\begin{tabular}{|c|c|c|c|c|c|c|c|}
\hline \multirow[b]{2}{*}{ Autor } & \multicolumn{3}{|c|}{ Visível } & \multicolumn{3}{|c|}{ Não visível } & \multirow{2}{*}{$\begin{array}{l}\text { Total } \\
\text { geral }\end{array}$} \\
\hline & Biópsia & Citologia & Global & Biópsia & Citologia & Global & \\
\hline Ikeda ${ }^{(8,21)}(1974)$ & - & - & - & - & - & - & $95,0 \%$ \\
\hline Kvale et al. ${ }^{(9)}(1976)$ & $71,0 \%$ & $76,8 \%$ & $86,0 \%$ & $\begin{array}{l}30,0 \% * \\
50,0 \%\end{array}$ & $\begin{array}{l}27,8 \% * \\
40,0 \%\end{array}$ & $\begin{array}{l}46,6 \% * \\
80,0 \%\end{array}$ & $79,0 \%$ \\
\hline Popovich et al.(26) (1981) & $92,0 \%$ & $88,0 \%$ & $96,0 \%$ & $70,0 \%$ & $40,0 \%$ & $75,0 \%$ & $86,9 \%$ \\
\hline Lundgren et al. ${ }^{(18)}$ (1983) & $85,3 \%$ & $70,5 \%$ & $97,0 \%$ & $32,1 \%$ & $50,0 \%$ & $70,0 \%$ & $88,0 \%$ \\
\hline Mak et al. ${ }^{(20)}(1990)$ & $76,0 \%$ & $74,0 \%$ & $87,2 \%$ & $36,5 \%$ & $42,3 \%$ & $55,6 \%$ & $76,6 \%$ \\
\hline Tregnago et al. ${ }^{(8)}$ (1994) & $76,7 \%$ & $53,3 \%$ & $92,1 \%$ & $18,2 \% *$ & $39,4 \% *$ & $42,1 \% *$ & $72,1 \%$ \\
\hline Lorenzoni et al. (2000) & $94,2 \%$ & $43,7 \%$ & $96,2 \%$ & $72,7 \% *$ & $38,4 \% *$ & $63,8 \% *$ & $83,1 \%$ \\
\hline
\end{tabular}

* Sem fluoroscopia revisão de 676 casos, detectaram sinais diretos de tumor em $82,7 \%$. Tregnago et al. ${ }^{(8)}$, em sua casuística coletada também no Brasil, observaram tumor endobrônquico em $64 \%$ de sua amostra. Esse fato é fundamental na análise de sensibilidade, pois na maioria das séries da literatura, quando o tumor é visível, o rendimento da fibrobroncoscopia é sensivelmente maior (Tabela 6). Os sinais indiretos ou menos específicos de neoplasia, como compressão extrínseca, carinas rombas, irregularidade de mucosa, são freqüentes. A imensa maioria dos exames mostra alterações que sugerem o diagnóstico ou indicam o local da segmentação brônquica no qual os procedimentos endoscópicos devem ser realizados. Observa-se que sinais como hiperemia, secreção e edema não foram computados por sua alta inespecificidade.

Em nosso serviço, realizam-se lavado, escovado e biópsia das lesões em todos pacientes com suspeita de neoplasia pulmonar. O valor do exame citopatológico no diagnóstico de neoplasia pulmonar está bem estabelecido, sendo seu percentual de falso-positivos inferior a $1 \%(4,6,8,9,15)$. Como o processamento citopatológico da amostra se faz concomitantemente, não há custos adicionais em termos de material. Assim, optou-se por analisar a citologia do lavado e escovado brônquico em conjunto. Considerou-se diagnóstico definitivo de câncer a presença de células neoplásicas nos espécimes citológicos ou anatomopatológicos, uma vez que a literatura mostra boa correlação entre estes e o diagnóstico pela citologia é aceito pela unanimidade dos autores ${ }^{(6,8,9,15,24,25)}$.

A broncoscopia mostrou boa sensibilidade diagnóstica, $83,1 \%$ (74 em 89), semelhante à da literatura $(8,9,14,18,20,21,26)$ (Tabela 6).

No grupo 1 o diagnóstico foi de $96,2 \%$ dos pacientes, enquanto no grupo 2 foi de $63,8 \%$. O menor número de diagnóstico foi no grupo 2 , pelo fato de as lesões pulmonares serem mais periféricas neste grupo.

No grupo 1 a sensibilidade global do método para o diagnóstico de câncer foi muito boa $(96,2 \%)$ comparável à de vários autores $^{(8,9,18,20,21,26)}$, de $86 \%$ a $97 \%$ (Tabela 6).

A biópsia de lesão contribui com um percentual maior de positividade $(94,2 \%)$ do que a citologia (43,7\%). Esta tendência não se confirma na literatura, em que o rendimento dos dois métodos é aproximadamente comparável ${ }^{(8,9,18,20,21,26)}$ (Tabe- 
la 6). A razão para tal não é clara; a menor positividade da citologia ocorre provavelmente pelo fato de que em tumor endoscopicamente visivel dificilmente a biópsia é realizada. A combinação dos dois exames resulta em maior percentual de positividade $(96,2 \%)$, de maneira que realizamos rotineiramente o lavado, o escovado e a biópsia. Entretanto, esse percentual de positividade é atingido com técnica correta e através de um número adequado de biópsias.

No grupo 2 a sensibilidade da fibrobroncoscopia foi baixa $(63,8 \%)$. Os percentuais observados nas séries da literatura variaram de $42,1 \%$ a $75 \%$, sendo nitidamente menor que no grupo dos tumores visiveis ${ }^{(8,9,18,20,21,26)}$. Nestas séries verificamos que o procedimento de maior rendimento foi diferente para os vários autores; para uns, foi a biópsia, para outros, a citologia (Tabela 6). A positividade dos dois exames sempre é superior aos percentuais de cada um isoladamente. Na presente série realizamos 22 biópsias, na presença de sinais indiretos de tumor ou critérios incompletos de infiltração, sem fluoroscopia. Neste grupo a sensibilidade diagnóstica foi de $72,7 \%$. A positividade da biópsia pulmonar no presente estudo $(72,7 \%)$ foi maior do que a anteriormente descrita pela literatu$\mathrm{ra}^{(8,9,18,20,21,26)}$ (Tabela 6), o que sugere que a biópsia endobrônquica deve ser indicada quando o paciente apresentar sinais indiretos de tumor ou critérios incompletos de infiltração. O lavado e escovado brônquico, realizados nos segmentos presumíveis da lesão (localizados pela radiografia de tórax), não foram suficientes para melhorar a sensibilidade diagnóstica da nossa série, que foi de $38,4 \%$. Este valor está abaixo do da maioria das séries ${ }^{(8,9,18,20,21,26)}$. Não foram realizadas biópsias transbrônquicas nos pacientes pesquisados.

Radke et al. (27), em uma análise de 97 pacientes submetidos à fibrobroncoscopia orientada por fluoroscopia bidimensional, concluíram que o tamanho da lesão $(>2 \mathrm{~cm})$ foi o fator de maior importância na sensibilidade do método. Isto porque as lesões maiores são ventiladas por maior número de brônquios e o seu acesso pelos instrumentos é mais fácil.

A realização de fibrobroncoscopia nesses pacientes não se acompanhou de "complicações graves" (pneumotórax, hemorragia pulmonar (> 50ml) e insuficiência respiratória). Complicações menores como broncoespasmo, sangramento brônquico e broncorréia ocorreram em 11,2\% dos pacientes; no grupo 1 ocorreram sangramento brônquico $(7,5 \%)$, broncoespasmo $(5,6 \%)$ e broncorréia $(1,8 \%)$. No grupo $2,27 \%$ tiveram sangramento brônquico e $2,7 \%$, broncoespasmo. Não houve nenhum caso de óbito.

\section{REFERÊNCIAS}

1. Mendes ESPS. Câncer de pulmão: novos horizontes. J Pneumol 1996;22:277-278.
2. Wünsch VF. Riscos ocupacionais e câncer de pulmão. J Pneumol 1995;21:34-42.

3. Tadokoro H. Câncer de pulmão: considerações sobre 300 casos. (Tese de Doutorado. Escola Paulista de Medicina). São Paulo. 1992. 188p.

4. Guimarães MJB. Câncer de pulmão - Diagnóstico e estadiamento. J Pneumol 1987;13:109-118.

5. Governo do Estado de Mato Grosso do Sul. Secretaria de Coordenação e Planejamento. Relatório Preliminar de Mortalidade Proporcional por Causa no Estado de Mato Grosso do Sul. Campo Grande, Sistema de Informações sobre Mortalidade, 1998.

6. Jett J, Feins R, Kvale P, McCloud T, et al. Pretreatment evaluation of nonsmall-cell lung cancer. Am J Respir Crit Care Med 1997;156:320-332.

7. Zamboni M. O novo estadiamento do câncer de pulmão. J Pneumol 1998;24:1-2.

8. Tregnago R, Grossman G, Leite RS, Matias M, Dornelles E, Xavier R. Contribuição da fibrobroncoscopia no diagnóstico do carcinoma brônquico. J Pneumol 1994;20:24-28.

9. Kvale PA, Bode FR, Kini S. Diagnostic accuracy in lung cancer. Chest 1976;69:752-757.

10. Govert JA, Kopita JM, Matchar D, Kussin PS, Samuelson WM. Costeffectiveness of collecting routine cytologic specimens during fiberoptic bronchoscopy for endoscopically visible lung tumor. Chest 1996;109:451-456.

11. Kamholz SL. Fiberoptic bronchoscopy. Chest 1996;109:593-594.

12. Minami H, Ando Y, Nomura F, Sakai S, Shimokata K. Interbronchoscopist variability in the diagnosis of lung cancer by flexible bronchoscopy. Chest 1994;105:1658-1662.

13. Poe RH, Levy PC, Israel RH, Ortiz CR, Kallay MC. Use of fiberoptic bronchoscopy in the diagnosis of bronchogenic carcinoma. Chest 1994;105:1663-1667.

14. Parada AA, Tassi EPC. Broncoscopia. J Pneumol 1986;12:249-261.

15. Prolla JC. Citopatologia do aparelho respiratório. J Pneumol 1986;12:98-206

16. Tonietto V, Lopes CA, Dias CF, Pilz FJ. Carcinoma brônquico e tabagismo: análise de 667 casos. J Pneumol 1992;18:23-27.

17. Chechani V. Bronchoscopic diagnosis of solitary pulmonary nodules and lung masses in the absence of endobronchial abnormality. Chest 1996; 109:620-625.

18. Lundgren R, Bergman F, Angstron T. Comparison of transbronchial fine needle aspiration biopsy, aspiration of bronchial secretion, bronchial washing, brush biopsy and forceps biopsy in the diagnosis of lung cancer. Eur J Respir Dis 1983;64:378-385.

19. Uehara C, Jamnik S, Santoro IL. Câncer de pulmão. Medicina, Ribeirão Preto 1998;31:266-276.

20. Mak VHF, Johnston IDA, Hetzel RM, Grubb C. Value of washings and brushings at fibreoptic bronchoscopy in the diagnosis of lung cancer. Thorax 1990;45:373-376.

21. Ono R, Loke J, Ikeda S. Bronchofiberscopy with curette biopsy and bronchography in the evalution of peripheral lung lesions. Chest 1981;79:162-166.

22. Shure D, Astarita RW. Bronchogenic carcinoma presenting as an endobronchial mass. Chest 1983;83:865-867.

23. Costa MJM, Silva NH, Madi K, Lima FPS, Carvalho MGC. Expressão da p53 nos tumores de pulmão de apresentação central e periférica. J Pneumol 1996;22:295-298.

24. Pirozynski M. Bronchoalveolar lavage in the diagnosis of peripheral, primary lung cancer. Chest 1992;102:372-374.

25. De Gracia J, Brave C, Miravitlles M, et al. Diagnostic value of bronchoalveolar lavage in peripheral lung cancer. Am Rev Respir Dis 1993; 147:649-652.

26. Popovich HR, Kvale PA, Eichenhorn MS, et al. Diagnostic accuracy of multiple biopsies from flexible fiberoptic bronchoscopy. Am Rev Respir Dis $1982 ; 125: 521-523$.

27. Radke JR, Conway WA, Eyler WR, Kvale PA. Diagnostic accuracy in peripheral lung lesions. Chest 1979;76:176-179. 